\title{
Elevated DHODH expression promotes cell proliferation via stabilizing $\beta$-catenin in esophageal squamous cell carcinoma
}

\author{
Yu Qian ${ }^{1,2}$, Xiao Liang ${ }^{2}$, Pengzhou Kong ${ }^{2}$, Yikun Cheng ${ }^{1}$, Heyang Cui ${ }^{1,2}$, Ting Yan², Jinghao Wang ${ }^{3}$, Ling Zhang ${ }^{1,2}$, \\ Yiqian Liu', Shiping $\mathrm{Guo}^{4}$, Xiaolong Cheng ${ }^{2}$ and Yongping Cui ${ }^{1}$
}

\begin{abstract}
As a key enzyme in de novo pyrimidine biosynthesis, the expression level of dihydroorotate dehydrogenase (DHODH) has been reported to be elevated in various types of malignant tumors and its tumor-promoting effect was considered to relate to its pyrimidine synthesis function. Here, we revealed one intriguing potential mechanism that DHODH modulated $\beta$-catenin signaling in esophageal squamous cell carcinoma (ESCC). We demonstrated that DHODH directly bound to the $\mathrm{NH} 2$ terminal of $\beta$-catenin, thereby, interrupting the interaction of GSK3 $\beta$ with $\beta$-catenin and leading to the abrogation of $\beta$-catenin degradation and accumulation of $\beta$-catenin in the nucleus, which in turn, resulted in the activation of $\beta$-catenin downstream genes, including CCND1, E2F3, Nanog, and OCT4. We further demonstrated that the regulation of $\beta$-catenin by $\mathrm{DHODH}$ was independent of $\mathrm{DHODH}$ catalyzing activity. Univariate and multivariate analyses suggested that DHODH expression might be an independent prognostic factor for ESCC patients. Collectively, our study highlights the pivotal role of DHODH mediated $\beta$-catenin signaling and indicates that $\mathrm{DHODH}$ may act as a multi-functional switcher from catalyzing pyrimidine metabolism to regulating tumor-related signaling pathways in ESCC.
\end{abstract}

\section{Introduction}

Esophageal squamous cell carcinoma (ESCC) is the ninth leading cause of cancer-related death worldwide, with over 515,000 new cases annually ${ }^{1-3}$. China has the highest incidence and mortality rate of ESCC; $70 \%$ of ESCC occur in China ${ }^{4,5}$. The 5-year survival rate of ESCC tends to be poor due to the lacking of early diagnosis, limited therapeutic options and frequent relapse ${ }^{6,7}$. Therefore, efforts are needed to explore the molecular

\footnotetext{
Correspondence: Xiaolong Cheng (chengx|@sxmu.edu.cn) or

Yongping Cui (cuiyp@sphmc.org)

${ }^{1}$ Cancer Institute, Peking University Shenzhen Hospital, Shenzhen Peking University-Hong Kong University of Science and Technology (PKU-HKUST) Medical Center, 518035 Shenzhen, People's Republic of China

${ }^{2}$ Department of Pathology \& Shanxi Key Laboratory of Carcinogenesis and

Translational Research on Esophageal Cancer, Shanxi Medical University, 030001 Taiyuan, Shanxi, People's Republic of China

Full list of author information is available at the end of the article

These authors contributed equally: Yu Qian, Xiao Liang, Pengzhou Kong

Edited by G. Ciliberto
}

mechanism underlying ESCC tumorigenesis for identification of molecular markers for diagnosing or predicting patient outcomes.

Dihydroorotate dehydrogenase (DHODH) is the ratelimiting enzyme in the uridine monophosphate (UMP) biosynthetic pathway and mostly located in the inner membrane of mitochondria, catalyzing the transformation of dihydroorotate to orotate dependent of $\mathrm{Mg}^{2+}$ and $\mathrm{ATP}^{8,9}$. Mutations of $\mathrm{DHODH}$ have been associated with various genetic diseases. For example, DHODH G202A, R346W causes deficient protein stability and R135C impairs the enzymatic activity, which are linked to Miller syndrome $^{10,11}$. DHODH polymorphism was reported to be linked with rheumatoid arthritis and lung cancer as well $^{12-14}$. Additionally, enhanced activity of DHODH has been implicated as a biomarker of malignant tumor including gastric cancer and skin cancer ${ }^{15,16}$. Ultraviolet radiation could transcriptionally induce DHODH

\section{(c) The Author(s) 2020}

(c) (i) Open Access This article is licensed under a Creative Commons Attribution 4.0 International License, which permits use, sharing, adaptation, distribution and reproduction c. in any medium or format, as long as you give appropriate credit to the original author(s) and the source, provide a link to the Creative Commons license, and indicate if changes were made. The images or other third party material in this article are included in the article's Creative Commons license, unless indicated otherwise in a credit line to the material. If material is not included in the article's Creative Commons license and your intended use is not permitted by statutory regulation or exceeds the permitted use, you will need to obtain permission directly from the copyright holder. To view a copy of this license, visit http://creativecommons.org/licenses/by/4.0/. 
expression by activating STAT3 ${ }^{17}$. Inhibition of DHODH leads to the starvation of pyrimidine pool and thereby decreases DNA and RNA synthesis and cell proliferation $^{18,19}$. Meanwhile, DHODH has impacts on mitochondrial Oxidative phosphorylation (OXPHOS), which is also responsible for de novo pyrimidine synthesis pathway $^{20}$. However, little is known about the biological function of $\mathrm{DHODH}$ and molecular mechanism responsible for ESCC.

In this study, we report that DHODH may directly interact with $\beta$-catenin and mediate $\beta$-catenin stabilization by interpreting its phosphorylation, thus prevent the polyubiquitination and induce $\beta$-catenin nuclear translocation, leading to ESCC cells proliferation and tumorigenesis. Furthermore, we demonstrated that DHODH was upregulated in ESCC samples compared with adjacent normal tissues and high expression of DHODH was significantly associated with early stages and shorter patient survival in ESCC.

\section{Materials and methods Clinical samples}

This study was approved by the Ethics Committee of Shanxi Medical University (Approval No.2017LL108). The written informed consents were received from all participates. ESCC Tissue microarrays (TMAs) were obtained from Shanghai Outdo Biotechnology Company Ltd. One TMA includes 38 cases of ESCC, 7 cases of carcinoma in situ, 22 cases of atypical hyperplasia, and 10 cases of adjacent non-cancer tissues. The other TMA contains 208 cases of ESCC tissues and non-cancer tissues with clinicopathological parameters (male 162 cases, female 46 cases, age $45-92$ years, median age 67 years, 2 to 9-year follow-up).

\section{Cell lines, reagents, and antibodies}

ESCC cell lines KYSE140, KYSE150, KYSE180, KYSE410, KYSE510, KYSE450, KYSE680, ECA109 and immortalized esophageal epithelial cell SHEE were generous gifts from Dr. Qimin Zhan (Key laboratory of Carcinogenesis and Translational Research, Peking University Cancer Hospital \& Institute) and stored at Shanxi Key Laboratory of Carcinogenesis and Translational Research on Esophageal Cancer, Shanxi Medical University. Cells were authenticated and tested as mycoplasma free. Cells were grown in RPMI1640 media (Hyclone, USA) supplementary with $10 \%$ fetal bovine serum (FBS), $100 \mathrm{U} / \mathrm{ml}$ penicillin, and $100 \mu \mathrm{g} / \mathrm{ml}$ streptomycin.

Detailed reagents and antibodies used in this study were described in the Supplementary Table 1. The lentiviruses encoding two different short-hairpin RNA (shRNA) sequences specific against $\mathrm{DHODH}$ (sequence from Sigma-Aldrich) were purchased from Cyagen Biosciences
Inc. After confirming the knocking down efficiency, two shRNAs were used as a pool. DHODH cDNA was cloned into pcDNA3.1 by BamH I and Xba I using primers as shown in Supplementary Table 2.

\section{Animal experiments}

Mice study was proceeded according to the guide for the Care and Use of Laboratory Animals in Shanxi Medical University. Mouse xenograft assay was performed on 4- to 6-week-old male BALB/c nude mice, and each group has 6 mice according to the guidelines. In all, $3 \times$ $10^{6}$ DHODH-overexpressing KYSE150 cells or DHODH knocking down ECA109 cells, were subcutaneously injected into the left oxter of nude mice, respectively. KYSE150 cells or ECA109 cells were used as control, respectively. After five weeks, mice were sacrificed and tumors were removed and measured. Tumor volumes were measured using caliper and calculated by the formula: length $(\mathrm{mm}) \times$ width $(\mathrm{mm})^{2} / 2$.

\section{Immunohistochemical staining (IHC)}

TMAs and serial $4 \mu \mathrm{m}$ paraffin-embedded sections from each xenograft tumor were subjected to IHC staining to evaluate the expression level of specific proteins. Briefly, the slides were deparaffinized in xylene, rehydrated in graded ethanol and treated with $3 \% \mathrm{H}_{2} \mathrm{O}_{2}$ to block endogenous peroxidase. Then antigen retrieval was performed in citrate buffer $\mathrm{pH} 6.0$ for $3 \mathrm{~min}$. After three washes with phosphate buffer saline (PBS), slides were incubated with primary antibodies at $4{ }^{\circ} \mathrm{C}$ overnight. After washing with PBS, the slides were incubated with MaxVision Mouse/Rabbit (MXB biotechnology, KIT-5020) and visualized with $\mathrm{DAB}$ and counterstained with hematoxylin. All images were captured by Aperio Scan Scope (AperioTechnology Inc, USA). Protein expression levels were analyzed using Aperio Cytoplasma 2.0 and Nuclear v.9 software.

\section{Cell proliferation assay}

Cell proliferation was measured by CCK- 8 assay and colony formation assay. For CCK-8 assay, 1500 indicated cells were seeded into 96-well plates. Ten microliters of CCK-8 solution (Boster, China) was added to each well and incubated at $37^{\circ} \mathrm{C}$ for $1 \mathrm{~h}$. The absorbance value was measured at $450 \mathrm{~nm}$. For colony formation assay, 2000 indicated cells were seeded into 6-well plates and cultured for 2 weeks and stained with $0.1 \%$ crystal violet.

\section{Flow cytometry}

For apoptosis analysis, cells were harvested, washed with PBS and binding buffer, then stained with Annexin V-FITC and PI using Annexin V-FITC/PI double staining kit (KeyGen Biotech) for $30 \mathrm{~min}$. Apoptotic cells were detected by flow cytometry using BD Calibur. For cell 
cycle, cells were suspended in pre-cold $70 \%$ ethanol, then washed with PBS, incubated with RNase A, and stained with PI using Cell cycle detection kit (KeyGen Biotech). Cell cycle proportion was measured using BD Calibur.

\section{Immunofluorescence}

Cells were cultured on the coverslips and stained with MitoTracker DeepRed FM (Cellsignaling Technology) to indicate mitochondria according to the manufacture's protocol. Then, cells were permeabilized with $0.25 \%$ Triton X-100/PBS. Post $30 \mathrm{~min}$, cells were incubated with $0.5 \% \mathrm{BSA}$ for $1 \mathrm{~h}$ and primary antibodies overnight. Then, cells were washed and incubated with Alexa Fluor-labeled secondary antibodies (Invitrogen). The nuclear were counterstained using DAPI. High-resolution images were captured using the Olympus Fluoview 1000 microscope.

\section{RNA extraction and quantitative real-time PCR}

Total RNA was extracted using RNAiso Kit (Takara) and first-strand cDNA was synthesized using PrimeScript RT Master Kit according to the protocol. Real-time PCR was performed by SYBR Green methods (Takara) on StepOne plus real-time PCR system (Applied Biosystem). The gene expression was measured by $-2^{\Delta \Delta C T}$. $\beta$-actin was used as internal control. The primers used were shown in Supplementary Table 2.

\section{RNA-sequencing}

The DHODH knockdown KYSE150, KYSE180, ECA109 cells and matched control cells were subjected to RNA sequencing. Total RNA extraction and sequencing were performed by Annoroad Gene Technology (Beijing, China) with standard procedures. Briefly, total RNA was enriched by magnetic oligo- $\mathrm{d}(\mathrm{T})$ and fragmented, followed by reverse transcription with random hexamers. Then the secondary cDNA strain was synthesized and purified. After adapter ligation, the library was sequenced using Illumina Hiseq platform with the sequencing strategy PE150. Data analysis was performed by the TopHat-Cufflinks pipeline, and gene expression values were presented by FPKM (fragment per kilobase of transcript sequence per million mapped reads). Differential gene expressions were analyzed by DEGSeq method with $\log 2$ Ratio $\geq 1, q<0.05$ as cutoff value. The differential genes were clustered by gene set enrichment analysis according to Kyoto Encyclopedia of Genes and Genomes (KEGG) database.

\section{Luciferase reporter assay}

TOP/FOP plasmids were generous gift from Dr. Canhua Huang (Sichuan University). Cells were co-transfected with TOP flash/FOP flash luciferase report plasmid, pTKRL plasmid and DHODH plasmid or empty control for overexpression analysis while sh-DHODH and control
shRNA for knockdown analysis using Lipofectamine 2000 reagent. $48 \mathrm{~h}$ post transfection, cells were collected. The Top flash and Fop flash luciferase values were measured by TransDetect Double-Luciferase Reporter Assay Kit (TransGene, China) according to the manufacturer's protocol and normalized by Renilla luciferase activity.

\section{Western blot and immunoprecipitation (IP)}

Cells were lysed in RIPA buffer with protease inhibitor and phosphatase inhibitor cocktails (Roche). Total protein concentration was determined by BCA method. Sixty micrograms of total proteins were loaded into $10 \%$ sodium dodecyl sulfate polyacrylamide gel electrophoresis and then transferred onto polyvinylidene fluoride membranes (Millipore). The membranes were incubated with primary antibodies at $4{ }^{\circ} \mathrm{C}$ overnight. After washing with TBST, the membranes were incubated with IR-labeled secondary antibodies followed by visualizing using Licor Odyssey CLx. The relative protein quantification was performed by calculating the band intensity in ImageJ. For IP assay, cell lysates were incubated with antibodies overnight and Protein G Plus/Protein A agarose (Millipore) was added for $2 \mathrm{~h}$. The precipitation was boiled with SDS-loading buffer and analyzed by western bolt.

\section{Glutathione S-transferase (GST) pull-down}

Recombinant GST-tagged full-length DHODH and its truncated mutants containing an $\mathrm{N}$-terminal were expressed in E. coli BL21 (DE3) and induced with $0.1 \mathrm{mM}$ isopropyl $\beta$-d-1-thiogalactopyranoside (IPTG) for $16 \mathrm{~h}$. The bacteria were lysed by ultrasonic $(300 \mathrm{~W}, 8 \mathrm{~s}$ treatment, $8 \mathrm{~s}$ break for $20 \mathrm{~min}$ ) and debris was removed by centrifugation. The proteins were harvested by incubating with GST binding agarose beads at $4{ }^{\circ} \mathrm{C}$ for $2 \mathrm{~h}$. GST-pull down was performed by the total cell lysis incubation at $4{ }^{\circ} \mathrm{C}$ overnight and washing with $50 \mathrm{mM}$ Glutathione at $4^{\circ} \mathrm{C}$ for $2 \mathrm{~h}$, the supernatant was boiled with SDS-loading buffer and detected by western blot.

\section{Bio-layer interferometry binding assay}

The bio-layer interferometry assay was performed at $25^{\circ} \mathrm{C}$ on the Octet Red96 system (ForteBio) with $50 \mathrm{mM}$ Tris-HCL pH 8.0 as running buffer. Purified GSTDHODH proteins were loaded onto anti-GST coated biosensors for $5 \mathrm{~min}$ and the biosensors were equilibrated in the running buffer for $2 \mathrm{~min}$ to acquire the baseline. Then, the biosensors were incubated with various concentration of Flag- $\beta$-catenin proteins for $5 \mathrm{~min}$, followed by $5 \mathrm{~min}$ of dissociation. The data were analyzed and the binding parameters were calculated by software.

\section{Statistical analysis}

All statistical analyses were performed using the SPSS 21.0 software and graphs were visualized using the Prism 

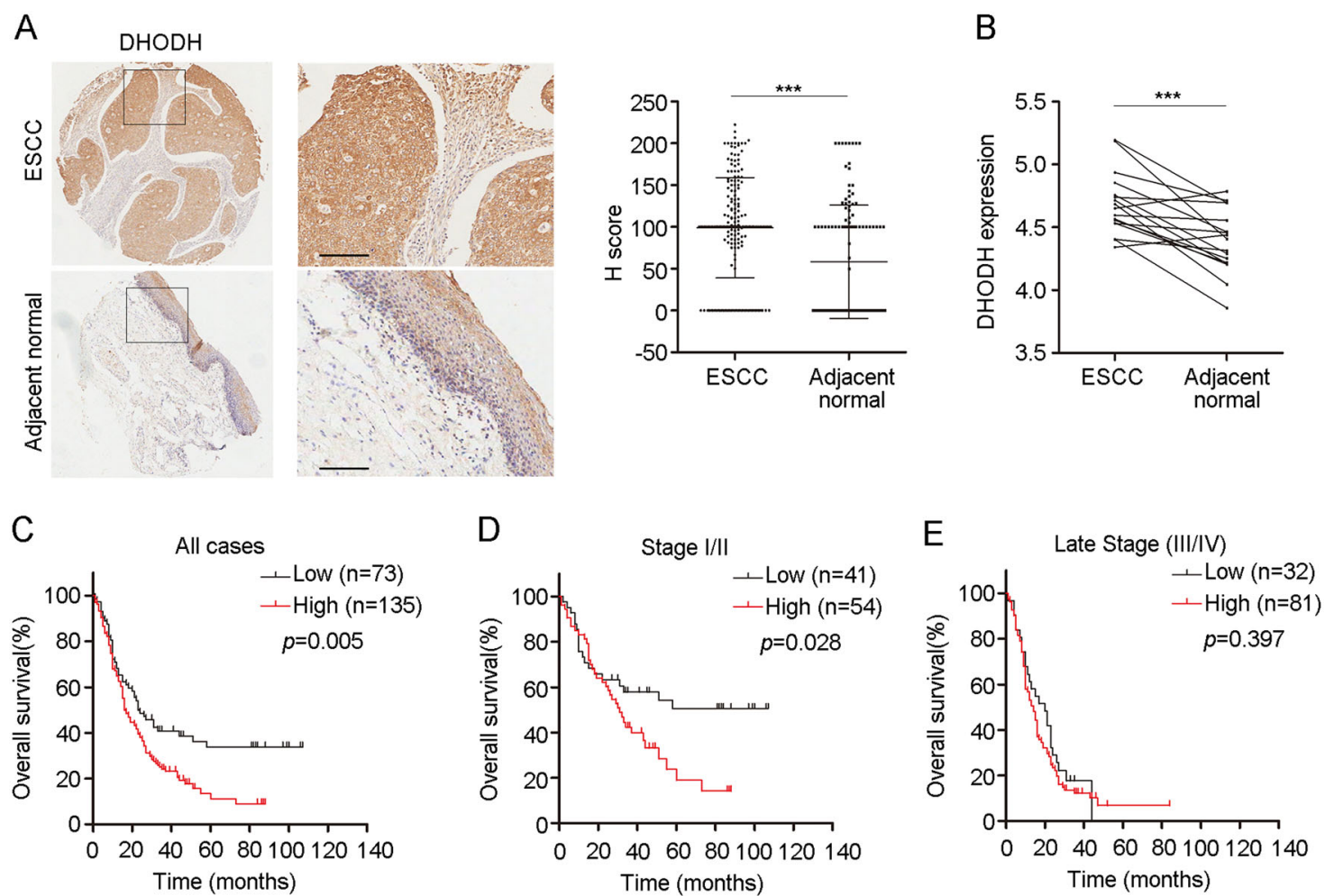

F
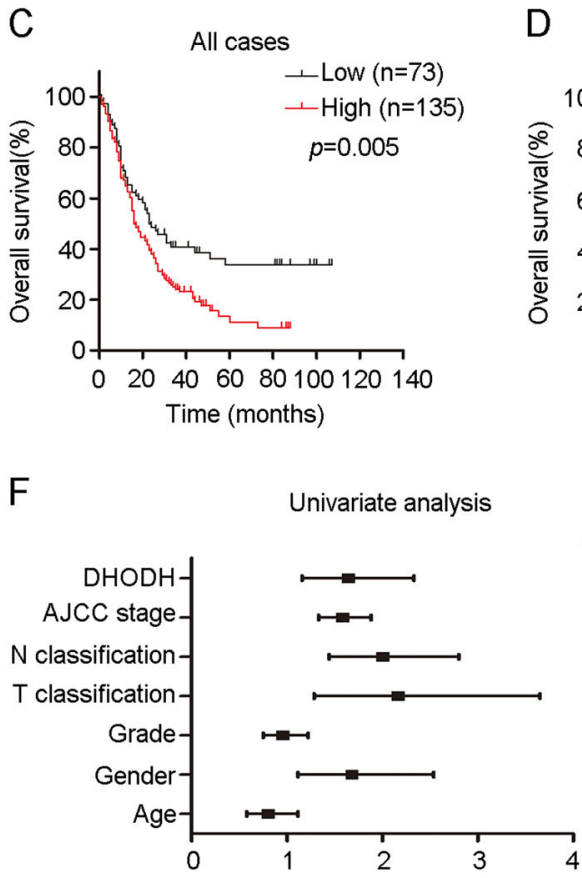

B

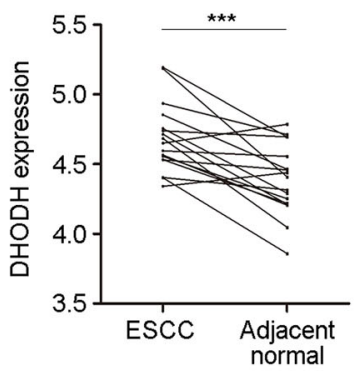

G

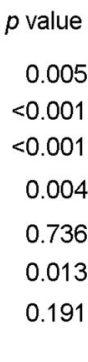

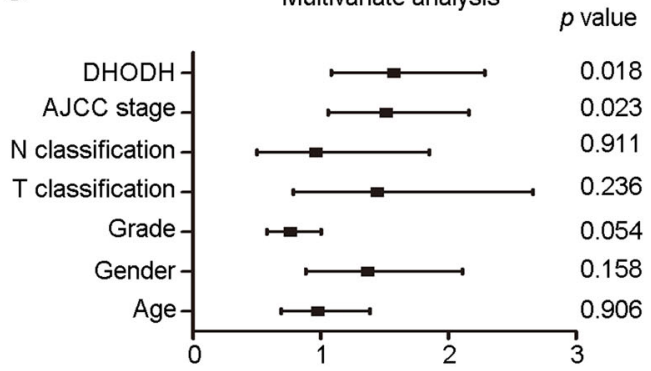

Fig. 1 DHODH overexpression is correlated with poor prognosis in ESCC. a The protein expression of DHODH was significantly higher in ESCC tissues compared with that in adjacent non-cancer tissues by IHC (left) and the scoring of IHC in 208 cases of ESCC (right). $\mathbf{b}$ mRNA expression of DHODH according to Hu's cohort. c Kaplan-Meier survival analysis of overall survival in 208 cases of ESCC patients. The receiver operating characteristic curve was used to determine the cutoff value. d-e Kaplan-Meier survival analysis of overall survival in stage I/II patients (d) and stage III/ IV patients (e). The univariate $(\mathbf{f})$ and multivariate $(\mathbf{g})$ cox regression analysis of DHODH expression and clinicopathological features of ESCC patients.

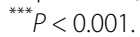

5 GraphPad. Data were presented as mean \pm SEM from at least three independent experiments. Differences between groups were measured by Student's $t$-test and ANOVA test. The expressions of DHODH in human tissues were compared by Wilcoxon signed-rank test. The correlation between DHODH level and clinicopathological features was analyzed by Chi-square test. Survival curves were constructed using the Kaplan-Meier method and differences in survival were evaluated using the log-rank test. Univariate and multivariate survival analyses were performed by a Cox proportional hazards regression model. The correlation between DHODH and $\beta$-catenin was measured by Pearson correlation coefficients. A $P$-value $<$ 0.05 was considered statistically significant.

\section{Results}

\section{High expression of DHODH was associated with poor prognosis of ESCC patients}

We performed IHC staining in TMAs containing tumor samples and matched normal adjacent tissues from 208 ESCC patients and found a predominant expression of DHODH protein in cytoplasm. DHODH level was significantly higher in tumors compared to that in adjacent normal tissues (Fig. 1a). We also found an elevation of 
Table 1 Associations between DHODH expression and clinicopathological parameters of ESCC patients.

\begin{tabular}{|c|c|c|c|c|c|}
\hline \multirow[t]{2}{*}{ Parameters } & \multirow[t]{2}{*}{ Case number } & \multicolumn{2}{|c|}{$\begin{array}{l}\text { DHODH } \\
\text { expression }\end{array}$} & \multirow[t]{2}{*}{$x^{2}$} & \multirow[t]{2}{*}{$P$} \\
\hline & & Low & High & & \\
\hline Overall & 208 & 73 & 135 & & \\
\hline \multicolumn{6}{|l|}{ Gender } \\
\hline Male & 162 & 54 & 108 & 0.68 & 0.41 \\
\hline Female & 46 & 19 & 27 & & \\
\hline \multicolumn{6}{|l|}{ Age (years) } \\
\hline$\leq 60$ & 70 & 25 & 45 & 0.03 & 0.853 \\
\hline$>60$ & 135 & 45 & 90 & & \\
\hline Unknown & 3 & 3 & 0 & & \\
\hline \multicolumn{6}{|c|}{ Tumor differentiation } \\
\hline Well & 48 & 22 & 26 & 4.26 & 0.119 \\
\hline Moderate & 120 & 41 & 79 & & \\
\hline Poor & 40 & 10 & 30 & & \\
\hline \multicolumn{6}{|c|}{ T classification } \\
\hline $\mathrm{T} 1-2$ & 29 & 14 & 15 & 1.94 & 0.164 \\
\hline T3-4 & 176 & 58 & 118 & & \\
\hline Unkown & 3 & 1 & 2 & & \\
\hline \multicolumn{6}{|c|}{ N classification } \\
\hline NO & 90 & 36 & 54 & 1.32 & 0.251 \\
\hline N1-2 & 118 & 37 & 81 & & \\
\hline \multicolumn{6}{|l|}{ AJCC stage } \\
\hline | and || & 95 & 41 & 54 & 4.36 & $0.037^{*}$ \\
\hline III and IV & 113 & 32 & 81 & & \\
\hline \multicolumn{6}{|l|}{ Status } \\
\hline Living & 52 & 28 & 24 & 9.63 & $0.002^{*}$ \\
\hline Death & 156 & 45 & 111 & & \\
\hline
\end{tabular}

DHODH mRNA in tumors according to Hu's cohort (Fig. $(\mathrm{b})^{21}$. Consistently, we observed a marked relative increase of DHODH expression in atypical hyperplasia compared with that of normal tissues, indicating the overexpression of DHODH may play an important role in the initiation of stage of ESCC (Supplementary Fig. S1A, B).

In addition, high DHODH expression was statistically associated with advanced stage (Table 1). In particular, we noticed that patients with high DHODH expression exhibited a worse prognosis (Fig. 1c). However, we found that DHODH expression was correlated with the poor survival of early stage (I and II) patients rather than late stage (III and IV) patients (Fig. 1d-e). Moreover, univariate Cox regression analyses showed that high DHODH expression, gender, $\mathrm{T}$ classification, $\mathrm{N}$ classification, and AJCC stage was associated with unfavorable overall survival, respectively. Further multivariate Cox regression analysis confirmed the AJCC stage and DHODH expression as potential independent prognostic factors (Fig. 1f-g and Table 2). Collectively, these results suggest that $D H O D H$ may be a potential predictor to estimate patients' survival.

\section{DHODH promotes cell proliferation and tumor growth in ESCC}

To discover the biological function of $D H O D H$ in ESCC, we detected the endogenous expression of DHODH in multiple ESCC cell lines (Supplementary Fig. S1C). We established stably DHODH-overexpressing KYSE510 and KYSE150 cell lines, and knockdown KYSE150, KYSE180 and ECA109 cell lines, respectively (Supplementary Fig. S1D, E). We found that DHODH significantly promoted cell proliferation as shown by CCK-8 assay (Fig. 2a-b) and colony formation assay (Fig. 2c-d). Additionally, leflunomide (Lef), a DHODH inhibitor, exerted a suppressive effect on cell proliferation as well (Supplementary Fig. S1F). Next, we turned to a xenograft mouse model and observed that mice baring DHODH-expressing KYSE150 cells formed malignant tumors more rapidly than that of control cells (Fig. 2e-f), that was confirmed by Ki67 staining assay (Fig. 2g). On the contrary, efficient DHODH deletion significantly prevented tumor growth (Fig. $2 \mathrm{~h}-\mathrm{j}$ ). Altogether, these data suggest that DHODH may act as an oncogene via promoting cell proliferation and tumor growth in ESCC.

\section{Inhibition of DHODH impedes cell cycle and induces apoptosis in ESCC cells}

Then we explored the effect of DHODH on cell cycle and apoptosis. Importantly, DHODH knockdown arrested cells in $\mathrm{S}$ phase and prolonged cell cycle as monitored by Live cell imaging (Supplementary Fig. S2A, B). Also, we found a remarkable decrease of cyclin $\mathrm{A}$ and cyclin $\mathrm{B}$ expression after DHODH knocking down (Supplementary Fig. 2C). DHODH knocking down induced apoptosis (Supplementary Fig. S2D). Meanwhile, overexpression of DHODH resulted in the opposite trends. We observed that DHODH inhibited apoptosis and promoted cell cycle (Supplementary Fig. S2E, F). By western blot, we analyzed the expression of pro-apoptosis marker BAX, antiapoptosis marker BCL2, and cell cycle markers such as E2F3, p53, and p21. Consistently, knockdown of DHODH increased BAX, p53 and p21 expression, which linked with increased apoptosis and cell cycle arrest. On the opposite, DHODH overexpression upregulated BCL2 and cell cycle related genes (Supplementary Fig. S2G, H). 
Table 2 Univariate and multivariate analysis for overall survival.

\begin{tabular}{|c|c|c|c|c|c|c|}
\hline \multirow[t]{2}{*}{ Parameters } & \multicolumn{2}{|c|}{ Univariate analysis } & \multirow[b]{2}{*}{$P$} & \multicolumn{2}{|c|}{ Multivariate analysis } & \multirow[b]{2}{*}{$P$} \\
\hline & HR & $95 \% \mathrm{Cl}$ & & HR & $95 \% \mathrm{Cl}$ & \\
\hline Age ( $\leq 60$ vs. $>60)$ & 0.802 & $0.577-1.116$ & 0.191 & 0.979 & $0.692-1.386$ & 0.906 \\
\hline Gender (female vs. male) & 1.681 & $1.114-2.538$ & $0.013^{*}$ & 1.371 & $0.885-2.122$ & 0.158 \\
\hline Histological differentiation (well vs. median vs. poor) & 0.959 & $0.752-1.223$ & 0.736 & 0.762 & $0.579-1.005$ & 0.054 \\
\hline T classification $(\mathrm{T} 1+2$ vs. $\mathrm{T} 3+4)$ & 2.165 & $1.284-3.651$ & $0.004^{*}$ & 1.446 & $0.785-2.664$ & 0.236 \\
\hline N classification (N0 vs. N1 + 2) & 2.01 & $1.442-2.802$ & $<0.001^{*}$ & 0.963 & $0.502-1.850$ & 0.911 \\
\hline AJCC stage $(I+\|$ vs. III + IV) & 1.583 & $1.331-1.882$ & $<0.001^{*}$ & 1.513 & $1.058-2.162$ & $0.023^{*}$ \\
\hline DHODH expression (low vs. high) & 1.644 & $1.158-2.333$ & $0.005^{*}$ & 1.573 & $1.083-2.287$ & $0.018^{*}$ \\
\hline
\end{tabular}

${ }^{*} P<0.05$

Hence, DHODH may play critical roles in the promotion of S-G2/M transition and the regulation of cell apoptosis.

\section{DHODH enhances Wnt/ $\beta$-catenin pathway in ESCC}

Then, we performed RNA-sequencing on DHODH knockdown KYSE150, KYSE180 and ECA109 cells, respectively (Fig. 3a and Supplementary Fig. S3A). By KEGG pathway enrichment analysis, we classified top significantly altered signaling pathways that were consistent from all three cell lines. Wnt/ $\beta$-catenin signaling was one of the most significantly enriched pathways (Fig. $3 \mathrm{~b}$ and Supplementary Fig. S3B, C). We noticed that the expressions of $\mathrm{Wnt} / \beta$-catenin target genes, such as CCND1, Oct4, and Nanog, were dramatically decreased after $D H O D H$ knockdown (Fig. 3c, d). Furthermore, we found $\mathrm{DHODH}$ knockdown reduced the transcriptional activity of $\beta$-catenin (Fig. 3e) using the dual luciferase reporter gene assay. Importantly, $\mathrm{DHODH}$ regulated $\beta$-catenin on protein expression while had no effect on the mRNA level (Fig. 3f and Supplementary Fig. S3D). As shown in Fig. 3f, knocking down of DHODH increased phospho- $\beta$-catenin ( $p-\beta$-catenin) level but decreased $\beta$-catenin as well as the downstream OCT4 and Nanog.

Inhibition of $\beta$-catenin signaling by XAV-939 caused restricted cell proliferation, even when DHODH was overexpressed (Fig. 3g). The expression of Oct4 also showed similar trend (Fig. 3h). Collectively, these results indicate that DHODH may act as a critical oncogene via activating Wnt/ $\beta$-catenin pathway in ESCC.

\section{DHODH stabilized $\beta$-catenin and promotes its nucleus translocation}

As $\mathrm{DHODH}$ regulated $\beta$-catenin at protein level, we examined the stability of $\beta$-catenin in $\mathrm{DHODH}$ overexpression or knockdown cells treated with cycloheximide $(\mathrm{CHX})$. We noticed that $\mathrm{DHODH}$ significantly stabilized $\beta$-catenin protein level (Fig. 4a, b). Consistently, the enhanced degradation of $\beta$-catenin was abrogated by bortezomib, an inhibitor of $26 \mathrm{~S}$ proteasome complex (Fig. $4 c)$. Moreover, DHODH knockdown reduced the nuclear localization of $\beta$-catenin (Fig. 4d).

We then investigated the relevance of $\mathrm{DHODH}$ and $\beta$-catenin in tumor samples. TMA analysis revealed a significant positive correlation between the expression level of DHODH and $\beta$-catenin (Fig. 4e and Supplementary Fig. S4A). Moreover, samples showing strong DHODH and $\beta$-catenin staining also exhibited strong nuclear staining of $\beta$-catenin (Fig. 4f). Moreover, patients exhibited low expression of both DHODH and $\beta$-catenin had longer overall survival than other patients (Fig. 4g).

This expression pattern was further confirmed in the slides of xenograft tumor sections derived from DHODH knockdown ECA109 cells and control cells (Supplementary Fig. S4B, C). These data suggest that DHODH was positively correlated with $\beta$-catenin at protein level in ESCC; and DHODH may involve in the regulation of $\beta$-catenin phosphorylation, thus affecting the degradation of $\beta$-catenin.

\section{DHODH binds the $\mathrm{NH} 2$ terminal of $\beta$-catenin and prevents its polyubiquitination}

We observed that DHODH localized at not only mitochondria but also nucleus, implying its unrevealed role in addition to catalyzing dihydroorotate (Supplementary Fig. S5A). DHODH was found to be co-localized with $\beta$-catenin by IF (Fig. 5a). Furthermore, endogenous $\beta$-catenin was readily detected in DHODH immunoprecipitates (Fig. 5b). Most importantly, bio-layer interferometry binding assay showed that the purified recombinant-DHODH protein directly bound to $\beta$-catenin, forming a 1:1 complex with a dissociation constant of $\sim 220 \mathrm{nM}$ (Fig. 5c). We constructed different domains of $\beta$-catenin including the $\mathrm{NH}_{2}$ terminal $(\mathrm{NH})$, the Armadillo repeat domain (AR), and the 


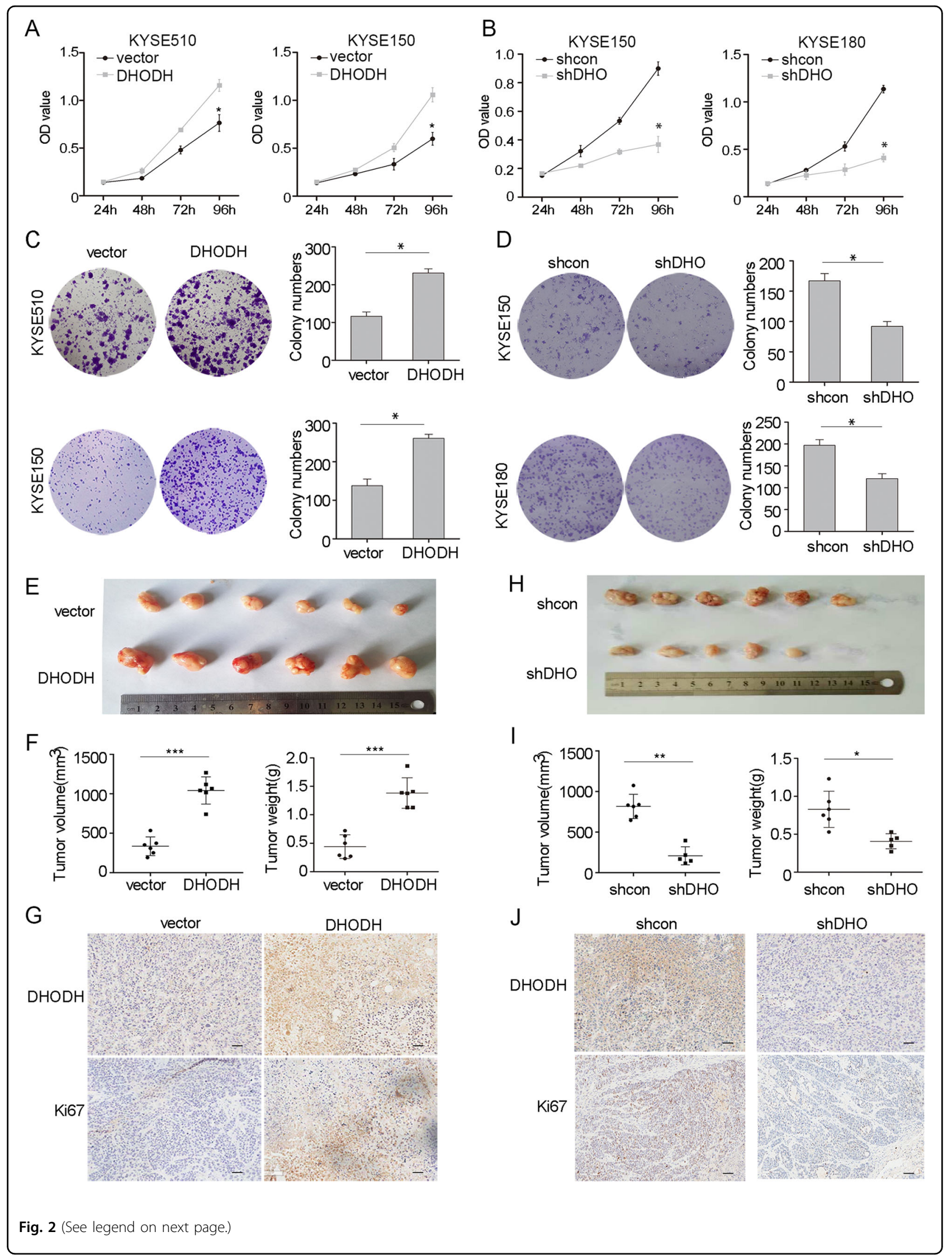


(see figure on previous page)

Fig. 2 DHODH induces tumorigenesis. a, b Cell proliferation assay was performed in DHODH overexpressed cells (KYSE510 and KYSE150, a), knocked down cells (KYSE150 and KYSE180, b), and correspondence control cells, respectively. c, d Colony formation assay was performed in DHODH overexpressed cells (c), DHODH knocked down cells (shDHO), and correspondence control cells (vector or shcon) (d). e Ectopic expressed DHODH in KYSE150 cells promoted tumor growth in xenograft mice model. $\mathbf{f}$ The volume and weight of tumors derived from DHODH ectopic expressed cells. $\mathbf{g}$ The expressions of DHODH and Ki67 were examined in tumor sections. $\mathbf{h}$ DHODH knockdown suppressed tumor growth in ECA109 cells. $\mathbf{i}$ The volume and weight of tumors derived from $\mathrm{DHODH}$ knocked down cells. $\mathbf{j}$ The expression of DHODH and Ki67 were examined (Bar, $100 \mu \mathrm{m}$ ). Data were representative of three independent experiments and are presented as mean $\pm \mathrm{SEM} .{ }^{*} P<0.05,{ }^{* *} P<0.01,{ }^{* * *} P<0.001$.

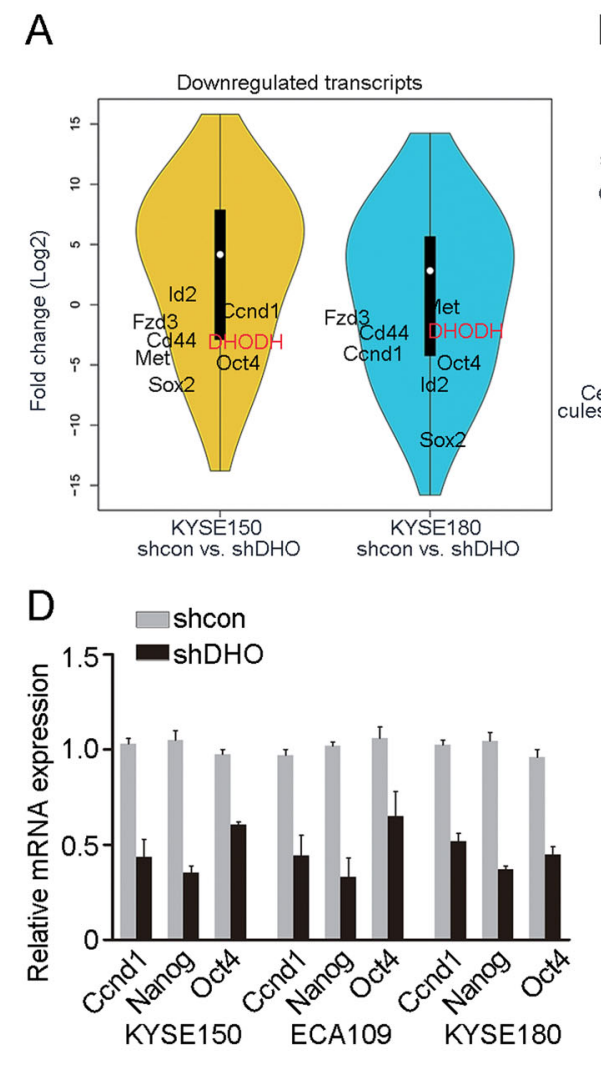

G

KYSE150

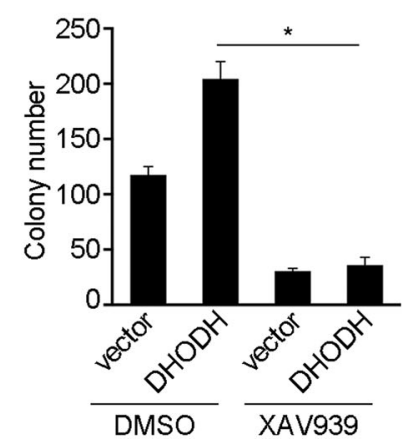

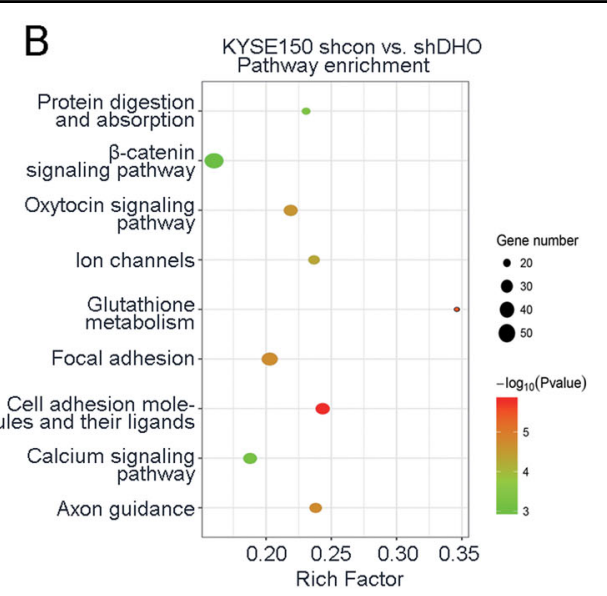

C

$\mathrm{E}$

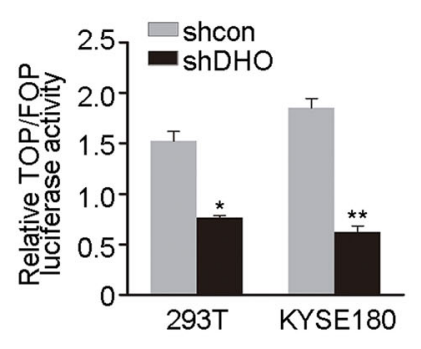

F

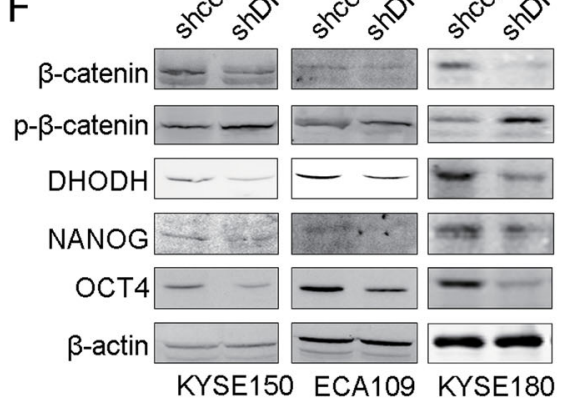

KYSE510

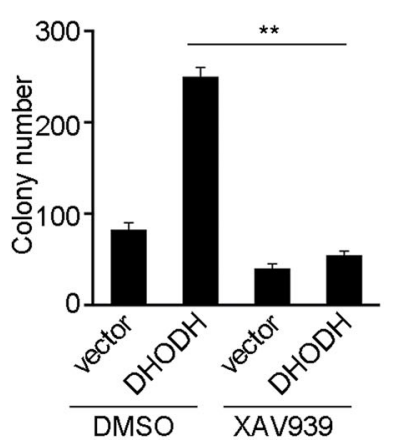

$\mathrm{H}$

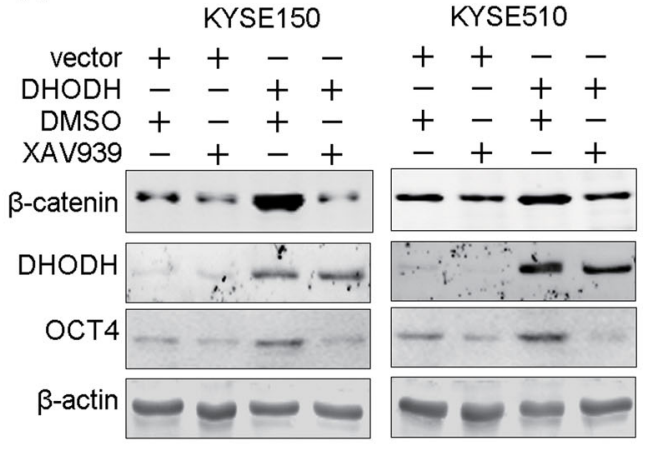

Fig. 3 DHODH activates $\beta$-catenin signaling. a Comparison of gene expression pattern between DHODH knockdown (shDHO) and control cells (shcon) in KYSE150 and KYSE180 cell lines by RNA-seq, respectively. Most of $\beta$-catenin related genes as well as DHODH were downregulated. b Pathway enrichment analysis showed that PI3K-Akt/ $\beta$-catenin pathway, protein digestion and absorption, ion channels were significantly enriched. c-d The expression levels of $\beta$-catenin regulated genes identified via RNA-seq (c) and qRT-PCR (d). e TOP/FOP flash assay depicted $\beta$-catenin downstream transcriptional activity was suppressed in DHODH knockdown 293T and KYSE180 cells. f Phospho- $\beta$-catenin, $\beta$-catenin, Nanog, and Oct4 were examined in DHODH knockdown and control KYSE150, KYSE180, and ECA109 cell lines, respectively. g Colony formation was detected after treated with XAV-939 in the control and DHODH overexpression cells. $\mathbf{h}$ XAV-939 impaired the upregulation of $\beta$-catenin and Oct4 by DHODH in KYSE510 and KYSE150 cells. Data were representative of three independent experiments and are presented as mean \pm SEM. ${ }^{*} P<0.05,{ }^{* *} P<0.01$. 

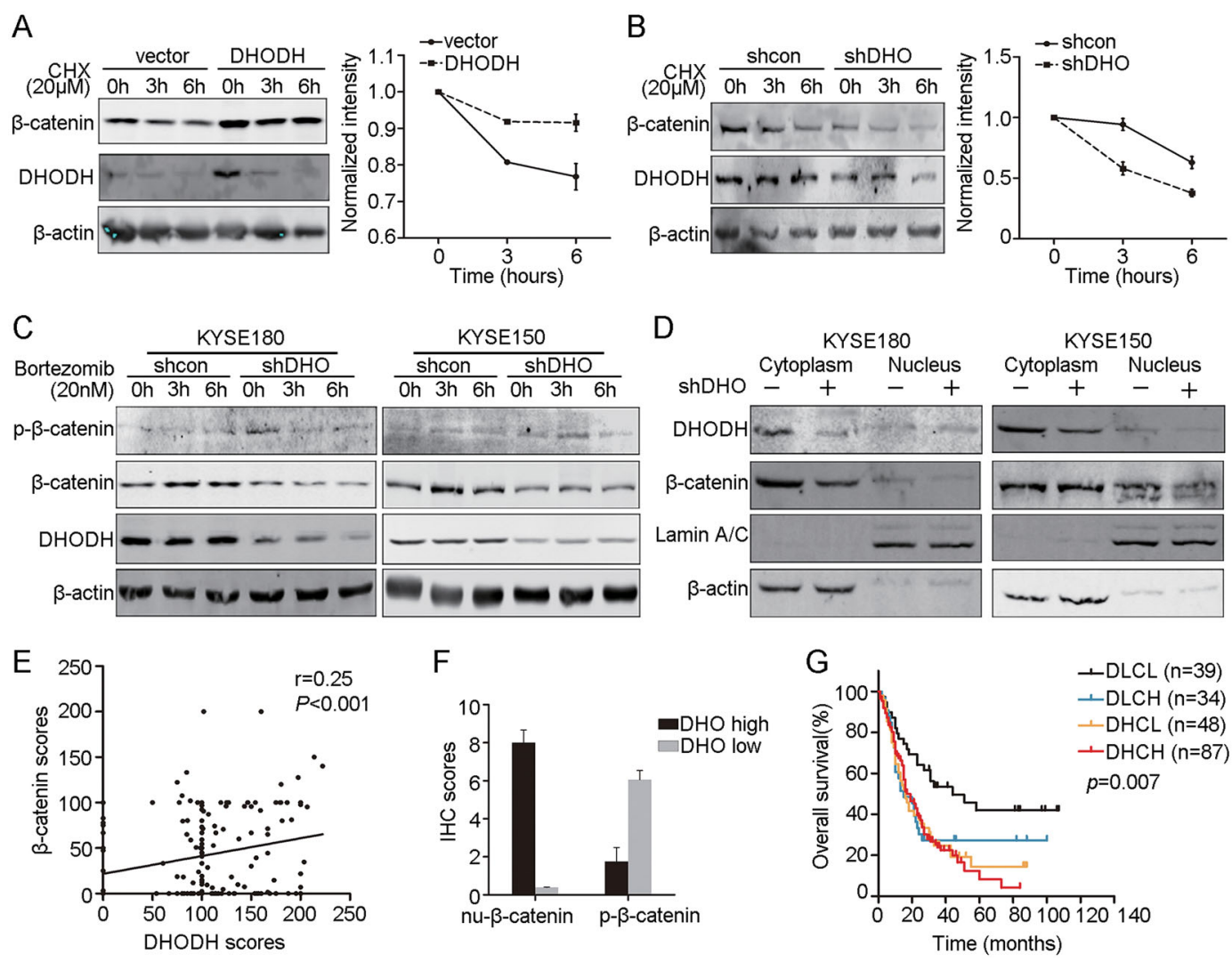

Fig. 4 DHODH mediates $\beta$-catenin stability and nuclear localization. a KYSE150 cells transfected with DHODH or vector were treated with CHX for the indicated times. $\mathbf{b}$ DHODH knockdown or control KYSE180 cells were treated with CHX for the indicated times. $\beta$-catenin expression was examined by western blot (left). A plot of normalized amount of $\beta$-catenin was shown (right). c DHODH knockdown or control KYSE150 and KYSE180 cells were treated with proteasome inhibitor, Bortezomib, for the indicated times. $\mathbf{d}$ DHODH knockdown decreased the cytoplasm and nucleus $\beta$-catenin expression. e The correlation of $\mathrm{DHODH}$ and $\beta$-catenin expression was examined using Prism 5. $\mathbf{f}$ The expression of nuclear $\beta$-catenin and phosphor- $\beta$-catenin were examined in DHODH high cases and low cases, respectively. $\mathbf{g}$ Kaplan-Meier analysis of patient's survival with $\beta$-catenin/ DHODH co-expression (DLCL, DHODH low/ $\beta$-catenin low, DLCH, DHODH low/ $\beta$-catenin high, DHCL, DHODH high/ $\beta$-catenin low, DHCH, DHODH high/ß-catenin high). ${ }^{*} P<0.05,{ }^{* *} P<0.01$.

transactivation domain (TD). As shown in Fig. 5d, HAtagged DHODH was observed in the Flag- $\beta$-catenin-NH and Flag- $\beta$-catenin full-length immunoprecipitates. In parallel experiments, $\beta$-catenin were specifically detected in GST-DHODH catalyze domain and full-length immunoprecipitates as revealed by GST-pull down assay (Fig. 5e and Supplementary Fig. S5B). These data suggest that the $\mathrm{NH}_{2}$ terminal $(\mathrm{NH})$ domain of $\beta$-catenin and the catalyze domain of DHODH are both required for the interaction.

We found that the level of polyubiquitin $\beta$-catenin complex was significantly increased in DHODH knockdown cells (Fig. 5f, g), as well as co-immunoprecipitated GSK3 $\beta$ level. Conversely, ectopic expression of DHODH decreased the ubiquitin level of $\beta$-catenin and GSK3 $\beta$ level in a dose-dependent manner (Fig. 5h). Given the fact that phosphorylation by GSK3 $\beta$ is critical for $\beta$-catenin stability, we used GSK3 $\beta$ inhibitor CHIR99021 and found that inhibition of GSK3 $\beta$ elevated the expression of $\beta$-catenin even DHODH was knocked down (Supplementary Fig. S5C).

Furthermore, we detected the catalytic dysfunctional DHODH-mut (R135C) and found that DHODHm could restore $\beta$-catenin expression, as well as wild-type DHODH (Supplementary Fig. S5D). Also, DHODHm promoted cell growth, cell cycle and inhibited apoptosis (Fig. 5i, j and Supplementary Fig. S5E, F).

\section{Discussion}

In this study, we found that DHODH overexpression promoted cell proliferation and tumor growth whereas DHODH knockdown caused reverse effects. However, the mechanism of DHODH mediated tumor progression was not fully investigated in ESCC.

$\beta$-catenin, first identified as a subunit of the cell to cell adhesion protein complex, acts critical role in the canonical Wnt Signaling. Targeting $\beta$-catenin activity and function could be therapeutic opportunities for many 


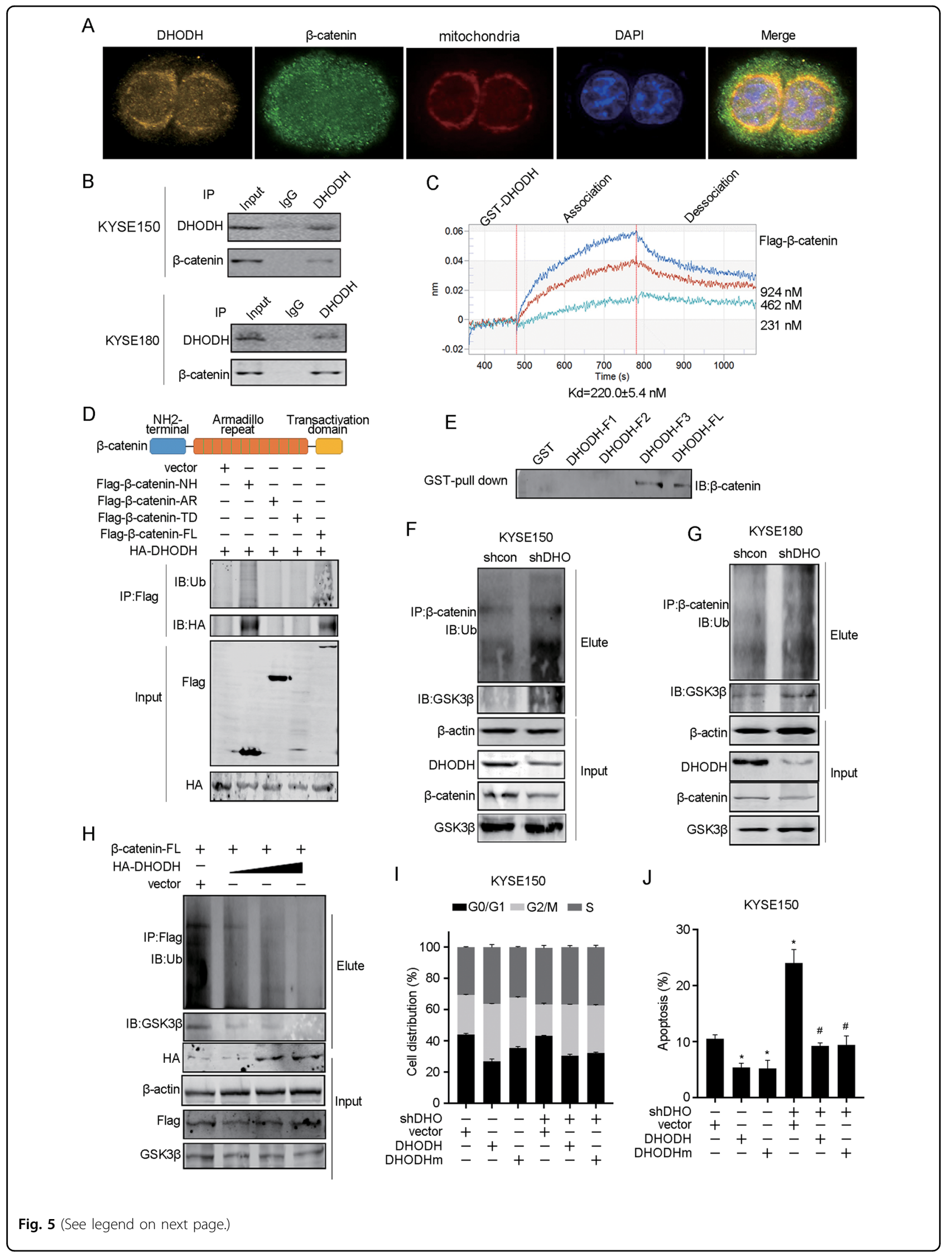


(see figure on previous page)

Fig. 5 DHODH interacts with $\beta$-catenin and prevents its polyubiquitination. a Immunofluorescent staining of DHODH, mitochondria and $\beta$-catenin. Nuclear DNA was visualized by DAPI staining. b Endogenous $\beta$-catenin was immunoprecipitated with anti-DHODH antibody or control lgG and analyzed by western blot in KYSE150 and KYSE180 cells. c Bio-layer interferometry assay of Flag- $\beta$-catenin binding to immobilized GST-DHODH. Kinetic analysis of the affinity of $\beta$-catenin and DHODH was performed. $\mathbf{d}$ A schematic diagram of different domains of $\beta$-catenin. Each domain was co-transfected into HEK-293T cells with DHODH, and the immunoprecipitated DHODH was examined by western blot. $\mathrm{NH}_{1} \mathrm{NH}_{2}$-terminal, $\mathrm{AR}$, Armadillo repeat, TD, Transactivation domain, FL, full-length. e GST-pull down assay was performed and the binding $\beta$-catenin was examined by western blot. $\mathbf{f}, \mathbf{g}$ The polyubiquitination of $\beta$-catenin was examined in DHODH knockdown KYSE150 (f) and KYSE180 (g) cells. $\mathbf{h}$ The polyubiquitination of $\beta$-catenin was examined after transfecting DHODH in gradient amount. i KYSE150 knocking down of DHODH cells were transfected with $\mathrm{DHODH}$ wild type or $\mathrm{DHODH}$ functional mutation $(\mathrm{DHODHm})$, respectively. Cell cycle distribution was examined. $\mathbf{j}$ Apoptosis assay of the abovementioned cells. Data were representative of three independent experiments and are presented as mean \pm SEM. ${ }^{*} P<0.05$, compared to control group; ${ }^{\# P}<0.05$, compared to shDHO alone group.

types of cancer, including ESCC ${ }^{22-24}$. Recently, numerous crosstalk with $\beta$-catenin were identified, including important pathways, non-coding RNAs, and metabolites $^{25-27}$. For example, Pyk 2 could decrease $p-\beta$-catenin and stabilize $\beta$-catenin, while PRL-3 activates $\beta$-catenin signaling pathway through Leo1 dephosphorylation in acute myeloid leukemia ${ }^{28,29}$. Canonical regulation of $\beta$-catenin linked with Wnt1 class ligand and receptors activation such as FZD and LRP5/LRP6 ${ }^{30}$. Destruction complex containing Axin, APC, $\mathrm{CK} 1 \alpha / \delta$, and $\mathrm{GSK} 3 \alpha / \beta$ couldnot phosphate $\beta$-catenin at several Ser/Thr site, and $\beta$-catenin will translocate into nuclear. Otherwise, $p$ $\beta$-catenin targeted by E3-ubiquitin ligase $\beta$-TrCP is degraded by ubiquitin-proteasome pathway ${ }^{31}$. However, Wnt5a type ligands or Wnt/Ca ${ }^{2+}$ acted as the noncanonical pathways regardless of $\beta$-catenin ${ }^{32,33}$. In our results, GSK3 $\beta$ inhibitor CHIR99021 could rescue the expression of $\beta$-catenin in DHODH knocking down cells. Therefore, our hypothesis would be activation of $\beta$-catenin pathway by DHODH via the way of effectiveness on phosphorylation of $\beta$-catenin state.

As a key enzyme in de novo pyrimidine biosynthesis, DHODH catalyzes the conversion of dehydration of dihydroorotate into orotate ${ }^{34}$. However, in the present study, we found that upregulation of $\beta$-catenin expression was triggered by catalyzing dysfunction form of $\mathrm{DHODH}$, suggesting that activating $\beta$-catenin pathway by $\mathrm{DHODH}$ was probably independent of its catalytic role in ESCC. Moreover, the interaction between GSK3 $\beta$ and $\beta$-catenin was partially abrogated after DHODH overexpression. Together, these data implied that $\mathrm{DHODH}$ promoted $\beta$-catenin stability by interrupting GSK3 $\beta$ mediated phosphorylation on the canonical pathway. Hence, inhibition of DHODH could impair $\beta$-catenin mediated tumorigenesis.

Many DHODH inhibitors have shown potential anticancer effects, such as ML390, brequinar, and chiral tetrahydroindazole $(\mathrm{HZO0})^{35-37}$. Some of them had been approved by FDA for acute myeloid leukemia ${ }^{38}$. Emerging evidence suggests that inhibition of DHODH impairs cancer cell proliferation, inducing differentiation. Also, downregulation of DHODH may have synergic effects together with inhibiting other oncogenes ${ }^{39-43}$. Of note, PTEN-mutant cancer was sensitive to DHODH inhibition, causing significant inadequate ATR activation and DNA damage ${ }^{44}$. Likewise, DHODH inhibition showed strong antitumor activity in mutant-KRAS-driven cancers $^{45}$. Targeting DHODH and disruption of the pyrimidine biosynthesis are demonstrated to be an approach to small cell lung cancer and colorectal cancer therapy $^{46,47}$. However, the molecular mechanisms of DHODH effecting on cancer development other than pyrimidine biosynthesis remains to be elucidated. We revealed that interaction between DHODH and $\beta$-catenin contributed to the alteration of malignancy phenotypes of ESCC. This may pave a way to probe a new molecular mechanism regarding to DHODH gene in cancer cell.

The clinical significance of our investigation revealed that patients with high expression of $\mathrm{DHODH}$ were predominant in early stage and had a much worse prognosis than ESCC patients with lower level. Therefore, detecting the expression level of $\mathrm{DHODH}$ may be a potential approach to early diagnosis or predicting outcomes for ESCC patient.

\section{Acknowledgements \\ This work was supported by funding from the National Natural Science Foundation of China $(81702779,81972613,81672768)$, the Key Laboratory of Shanxi Province (201705D111003), the Fund for Shanxi "1331 Project" and "1331 Project" Key Subjects Construction, the fund of "San-ming" Project of Medicine in Shenzhen (No. SZSM201812088), and the Guangdong Basic and Applied Basic Research Foundation (2019B030302012).}

\section{Author details}

${ }^{1}$ Cancer Institute, Peking University Shenzhen Hospital, Shenzhen Peking University-Hong Kong University of Science and Technology (PKU-HKUST) Medical Center, 518035 Shenzhen, People's Republic of China. ${ }^{2}$ Department of Pathology \& Shanxi Key Laboratory of Carcinogenesis and Translational Research on Esophageal Cancer, Shanxi Medical University, 030001 Taiyuan, Shanxi, People's Republic of China. ${ }^{3}$ Department of Obstetrics \& Gynecology, the Second Hospital, Shanxi Medical University, Taiyuan, Shanxi, China.

${ }^{4}$ Department of Tumor Surgery, Shanxi Cancer Hospital, Taiyuan, Shanxi, China

\section{Author contributions}

Y.P.C and X.L.C. supervised the study and revised the manuscript. Y.Q. conceived the study and wrote the manuscript. Y.Q., X.L., P.Z.K., T.Y., and Y.K.C. performed the experiments. H.Y.C, T.Y., Y.K.C. analyzed the RNA-seq data. J.H.W. 
and L.Z. performed the $\mathrm{IHC}$ data analysis and in vivo xenograft experiments. Y.Q.L. performed the flow cytometry. S.P.G. coordinated and performed pathology review. X.L.C. organized the administrative, technical, or material support.

\section{Conflict of interest}

The authors declare that they have no conflict of interest.

\section{Publisher's note}

Springer Nature remains neutral with regard to jurisdictional claims in published maps and institutional affiliations.

Supplementary Information accompanies this paper at (https://doi.org/ 10.1038/s41419-020-03044-1).

Received: 11 February 2020 Revised: 20 September 2020 Accepted: 22 September 2020

Published online: 15 October 2020

\section{References}

1. Bray, F. et al. Global cancer statistics 2018: GLOBOCAN estimates of incidence and mortality worldwide for 36 cancers in 185 countries. CA Cancer J. Clin. $\mathbf{6 8}$, 394-424 (2018).

2. Tungekar, A. et al. ESCC ATLAS: A population wide compendium of biomarkers for Esophageal Squamous Cell Carcinoma. Sci. Rep. 8, 12715 (2018).

3. Zeng, H. et al. Esophageal cancer statistics in China, 2011: Estimates based on 177 cancer registries. Thorac. Cancer 7, 232-237 (2016).

4. Gholipour, C., Shalchi, R. A. \& Abbasi, M. A histopathological study of esophageal cancer on the western side of the Caspian littoral from 1994 to 2003. Dis. Esophagus.: Off. J. Int. Soc. Dis. Esophagus. 21, 322-327 (2008).

5. Hu, X. et al. FAT1 prevents epithelial mesenchymal transition (EMT) via MAPK ERK signaling pathway in esophageal squamous cell cancer. Cancer Lett. 397, 83-93 (2017).

6. Talukdar, F. R. et al. Molecular landscape of esophageal cancer: implications for early detection and personalized therapy. Ann. New York Acad. Sci. https://doi. org/10.1111/nyas.13876 (2018).

7. Yuequan, J., Shifeng, C. \& Bing, Z. Prognostic factors and family history for survival of esophageal squamous cell carcinoma patients after surgery. Ann. Thorac. Surg. 90, 908-913 (2010).

8. Liu, S., Neidhardt, E. A., Grossman, T. H., Ocain, T. \& Clardy, J. Structures of human dihydroorotate dehydrogenase in complex with antiproliferative agents. Structure 8, 25-33 (2000)

9. Loffler, M., Fairbanks, L. D., Zameitat, E., Marinaki, A. M. \& Simmonds, H. A Pyrimidine pathways in health and disease. Trends Mol. Med. 11, 430-437 (2005).

10. Roach, J. C. et al. Analysis of genetic inheritance in a family quartet by wholegenome sequencing. Science 328, 636-639 (2010).

11. Fang, J. et al. Protein instability and functional defects caused by mutations of dihydro-orotate dehydrogenase in Miller syndrome patients. Biosci. Rep. $\mathbf{3 2}$ 631-639 (2012).

12. Pawlik, A. et al. The effect of exon $(19 C>A)$ dihydroorotate dehydrogenase gene polymorphism on rheumatoid arthritis treatment with leflunomide. Pharmacogenomics 10, 303-309 (2009).

13. O'Doherty, C. et al. Association of DHODH haplotype variants and response to leflunomide treatment in rheumatoid arthritis. Pharmacogenomics 13, 1427-1434 (2012).

14. Jones, C. C. et al. Cross-Cancer Pleiotropic Associations with Lung Cancer Risk in African Americans. Cancer Epidemiol. Biomark. Prev.: a Publ. Am. Assoc. Cancer Res., cosponsored Am. Soc. Preventive Oncol. 28, 715-723 (2019).

15. Yin, S., Kabashima, T., Zhu, Q., Shibata, T. \& Kai, M. Fluorescence assay of dihydroorotate dehydrogenase that may become a cancer biomarker. Sci. Rep. 7. 40670 (2017)

16. Hosseini, M. et al. Energy metabolism rewiring precedes UVB-induced primary skin tumor formation. Cell Rep. 23, 3621-3634 (2018).

17. Hosseini, M. et al. UVB-induced DHODH upregulation, which is driven by STAT3, is a promising target for chemoprevention and combination therapy of photocarcinogenesis. Oncogenesis 8, 52 (2019).
18. Buettner, R. et al. Leflunomide synergizes with gemcitabine in growth inhibition of PC cells and impairs c-myc signaling through PIM kinase targeting. Mol. Ther. Oncolytics 14, 149-158 (2019).

19. Echizenya, S. et al. Discovery of a new pyrimidine synthesis inhibitor eradicating glioblastoma-initiating cells. Neuro Oncol, https://doi.org/10.1093/ neuonc/noz170 (2019).

20. Bajzikova, M. et al. Reactivation of dihydroorotate dehydrogenase-driven pyrimidine biosynthesis restores tumor growth of respiration-deficient cancer cells. Cell Metab. 29, 399-416 e310 (2019).

21. $\mathrm{Hu}, \mathrm{N}$. et al. Genome wide analysis of DNA copy number neutral loss of heterozygosity (CNNLOH) and its relation to gene expression in esophageal squamous cell carcinoma. BMC Genomics 11, 576 (2010).

22. Li, M. Y. et al. Ribosomal S6 protein kinase 4 promotes radioresistance in esophageal squamous cell carcinoma. J. Clin. Invest. 130, 4301-4319 (2020).

23. Zhang, Z. et al. Inhibition of the Wnt/beta-catenin pathway overcomes resistance to enzalutamide in castration-resistant prostate cancer. Cancer Res. 78, 3147-3162 (2018)

24. Lee, E. et al. Inhibition of androgen receptor and beta-catenin activity in prostate cancer. Proc. Natl Acad. Sci. USA 110, 15710-15715 (2013).

25. DiRenzo, D. M. et al. A crosstalk between TGF-beta/Smad3 and Wnt/betacatenin pathways promotes vascular smooth muscle cell proliferation. Cell Signal 28, 498-505 (2016).

26. Li, J. et al. Glypican-1 promotes tumorigenesis by regulating the PTEN/Akt/ beta-catenin signaling pathway in esophageal squamous cell carcinoma. Digestive Dis. Sci. 64, 1493-1502 (2019).

27. Hung, M. H. et al. Canagliflozin inhibits growth of hepatocellular carcinoma via blocking glucose-influx-induced beta-catenin activation. Cell Death Dis. 10, 420 (2019).

28. Narendra Talabattula, V. A. et al. Non-canonical pathway induced by Wnt3a regulates beta-catenin via Pyk2 in differentiating human neural progenitor cells. Biochem. Biophys. Res. Commun. 491, 40-46 (2017).

29. Chong, P. S. Y. et al. Non-canonical activation of beta-catenin by PRL-3 phosphatase in acute myeloid leukemia. Oncogene 38, 1508-1519 (2019).

30. Janda, C. Y. et al. Surrogate Wnt agonists that phenocopy canonical Wnt and beta-catenin signalling. Nature 545, 234-237 (2017).

31. Nusse, R. \& Clevers, H. Wnt/beta-catenin signaling, disease, and emerging therapeutic modalities. Cell 169, 985-999 (2017).

32. Chae, W. J. \& Bothwell, A. L. M. Canonical and non-canonical Wnt signaling in immune cells. Trends Immunol. 39, 830-847 (2018).

33. Scholz, B. et al. Endothelial RSPO3 Controls Vascular Stability and Pruning through Non-canonical WNT/Ca(2+)/NFAT Signaling. Dev. Cell 36, 79-93 (2016).

34. Baumgartner, R. et al. Dual binding mode of a novel series of $\mathrm{DHODH}$ inhibitors. J. Med. Chem. 49, 1239-1247 (2006).

35. Madak, J. T., Cuthbertson, C. R., Chen, W., Showalter, H. D. \& Neamati, N. Design, synthesis, and characterization of brequinar conjugates as probes to study DHODH inhibition. Chemistry 23, 13875-13878 (2017).

36. Miyazaki, Y. et al. Selective cytotoxicity of dihydroorotate dehydrogenase inhibitors to human cancer cells under hypoxia and nutrient-deprived conditions. Front. Pharmacol. 9, 997 (2018).

37. Ladds, M. et al. A DHODH inhibitor increases p53 synthesis and enhances tumor cell killing by p53 degradation blockage. Nat. Commun. 9, 1107 (2018).

38. Christian, $\mathrm{S}$. et al. The novel dihydroorotate dehydrogenase (DHODH) inhibitor BAY 2402234 triggers differentiation and is effective in the treatment of myeloid malignancies. Leukemia https://doi.org/10.1038/s41375-019-0461-5 (2019).

39. Lewis, T. A. et al. Development of ML390: a human DHODH inhibitor that induces differentiation in acute myeloid leukemia. ACS Med. Chem. Lett. 7 1112-1117 (2016)

40. Zhao, E., Jiang, X. \& Cui, H. Bombyx mori Dihydroorotate Dehydrogenase: Knockdown Inhibits Cell Growth and Proliferation via Inducing Cell Cycle Arrest. Int. J. Mol. Sci. 19, https://doi.org/10.3390/ijms19092581 (2018).

41. White, R. M. et al. DHODH modulates transcriptional elongation in the neural crest and melanoma. Nature 471, 518-522 (2011).

42. Sykes, D. B. et al. Inhibition of dihydroorotate dehydrogenase overcomes differentiation blockade in acute myeloid leukemia. Cell 167, 171-186 e115 (2016).

43. Dorasamy, M. S., Choudhary, B., Nellore, K., Subramanya, H. \& Wong, P. F. Dihydroorotate dehydrogenase inhibitors target c-myc and arrest melanoma, myeloma and lymphoma cells at S-phase. J. Cancer 8, 3086-3098 (2017). 
44. Mathur, D. et al. PTEN regulates glutamine flux to pyrimidine synthesis and sensitivity to dihydroorotate dehydrogenase inhibition. Cancer Discov. 7 380-390 (2017).

45. Koundinya, $M$. et al. Dependence on the pyrimidine biosynthetic enzyme $\mathrm{DHODH}$ is a synthetic lethal vulnerability in mutant KRAS-driven cancers. Cell Chem. Biol. 25, 705-717 e711 (2018).
46. Li, L. et al. Identification of DHODH as a therapeutic target in small cel lung cancer. Sci. Transl. Med. 11, https://doi.org/10.1126/scitranslmed. aaw7852 (2019).

47. Yamaguchi, N. et al. PCK1 and DHODH drive colorectal cancer liver metastatic colonization and hypoxic growth by promoting nucleotide synthesis. Elife $\mathbf{8}$, https://doi.org/10.7554/eLife.52135 (2019). 